\title{
LA LIBERTAD DE LAS COSAS: REPENSAR EL CLINAMEN HOY ${ }^{1}$
}

\author{
Valentina Bulo Vargas \\ Universidad de Santiago de Chile. Chile
}

Resumen: Las tesis centrales que la materialidad tiene que ver con el horizonte que fija los límites de lo que llamamos cuerpo y que uno de sus rasgos medulares podría ser pensado como libertad. Este momento se corresponde con la lectura que realizan varios contemporáneos sobre el clinamen de Epicuro y Lucrecio, como Nancy, Derrida, Serres y Althusser.

Palabras clave: Clinamen - parénklisis - Epicuro - Nancy - Materialidad

\section{The freedom of things: rethinking the clinamen today}

Abstract: The central thesis is that materiality has to do with the horizon that fixes the limits of what we call a body, and that one of its core traits could be thought as freedom. This moment corresponds to the reading made on the clinamen of Epicurus and Lucretius by several contemporary thinkers such as Nancy, Derrida, Serres and Althusser.

Keywords: Clinamen - Parenklisis - Epicurus - Nancy - Materiality.

Recibido:16.12.2016 - Aceptado: 10.03.2017

1 Este escrito forma parte del Proyecto de Investigación Fondecyt Regular $\mathrm{n}^{\circ} 1150266$, "Materialidad del cuerpo y la diferencia en la ontología de Jean-Luc Nancy" del que Valentina Bulo es Investigadora Responsable. 
"En algún sentido, la piedra es libre"

(Nancy, 1996, 177)

\section{El problema de la materialidad}

uando hablamos de cuerpo, en general, estamos suponiendo
una idea de materialidad. Usualmente asociamos la
materialidad a aquello de lo que un cuerpo está hecho para indicar simplemente aquel lado más contingente o concreto de ese cuerpo, también hablamos de materialización para aludir a una cierta toma de consistencia de algo y es que el cuerpo necesita un modo de entender la materialidad; para ser más exactos, pensar la materialidad tiene que ver con el horizonte que fija los límites de eso que llamamos cuerpo.

El pensamiento contemporáneo, desde muy distintas líneas como la fenomenología, la biopolítica, los estudios de género y especialmente la filosofía de las ciencias, ha situado a los cuerpos como un problema de primer orden, pero poco se ha detenido en su materialidad, ha reformulado los cánones para pensar los cuerpos, principalmente cuestionando dualidades como alma-cuerpo o naturaleza-artificio, pero un cuestionamiento a fondo de los cuerpos supone repensar su materialidad.

En el presente texto nos haremos cargo de realizar un bosquejo conceptual de un aspecto de la materialidad de los cuerpos que retoma la idea de parénklisis trabajada por Epicuro y reformulada luego desde la idea de clinamen en Lucrecio y lo haremos de la mano de algunos filósofos que han pensado de un modo central el problema de los cuerpos, especialmente Jean-Luc Nancy. Primeramente indicaremos algunos trazos del horizonte de comprensión de los cuerpos que nos permitirán luego desplazarnos desde los cuerpos a su materialidad.

\section{De los cuerpos a su materialidad}

El primer trazo del horizonte de comprensión de los cuerpos radica en el entenderlos más desde su carácter relacional que sustancial. Podríamos decir que en el pensamiento contemporáneo existen ciertos filósofos que tienen un carácter "preposicional" en el sentido de que su pensamiento está marcado, más que por ciertos conceptos sustanciales o sustantivos, por ideas vinculadas a pre-posiciones. En este sentido, por ejemplo, el pensamiento de Heidegger pondrá el acento en un Entre, como figura de la diferencia ontológica y como prioridad de la relación diferencial "entre" el 
ser y el ente. De un modo similar, habría que decir que Nancy es el filósofo del "con"”; ambos ponen el acento en desplazar el sentido clásico de las cosas como un qué o como una sustancia y en ambos hay una relación que determina los relatos, pero Nancy, filósofo del cuerpo y del tocar pondrá el acento en cómo se vinculan unos cuerpos con otros, "los cuerpos no se definen, funcionan, se acoplan, se performativizan, hacen mundo; y en este sentido aquello que pasa entre un cuerpo y otro pesa más que uno y otro, los cuerpos son relacionales y modales, se trata de los modos de cada vez de los cuerpos, unos con otros"(Cf. Bulo, 2012, 108ss).

Desde este carácter modal y relacional de los cuerpos es que es necesario fundamentar su relacionabilidad, y pensamos que la materialidad de los cuerpos apunta a ello, pues se vincula al momento de determinación de los cuerpos y a aquello que excede su determinación y posibilita su relacionabilidad. Más adelante nos detendremos en este punto, pero aquí es importante dejar consignada la necesidad de proponer la materialidad como aquel momento que, en última instancia, da cuenta de las condiciones de posibilidad de la relación de unos cuerpos con otros, de su relacionabilidad. Por ello, como veremos, la materialidad de los cuerpos no puede reducirse a la determinación ya que los cuerpos no se entienden primeramente en forma sustancial, desde un qué determinado.

A partir de lo anterior podemos afirmar que los cuerpos no se sitúan completamente en el ámbito de lo dado ni de lo construido; en su texto "De la strucción" Nancy explicará con detalle una tesis que para nuestro asunto es clave: la escisión entre naturaleza y técnica está difuminada, es decir que, y este es el segundo trazo que enunciaremos, no hay una distinción tajante entre los cuerpos naturales y artificiales; "si la técnica da el sentido de 'naturaleza' a partir de la cual ella se construye y ella destruye al mismo tiempo, esto quiere decir que no es más del todo posible hablar de 'naturaleza', ni tampoco, por lo tanto, finalmente, de la 'técnica'" (Nancy, 2013, 31). Por ello Nancy propone hablar más que de una construcción de mundo, o de una deconstrucción, de una strucción que no es otra cosa que el mero amontonamiento de los cuerpos, el "conjunto no ensamblado", un montón de cuerpos unidos por su contigüidad. He aquí el sentido del "con" en Nancy: "la pura y simple yuxtaposición que no hace sentido"

2 He trabajado con detalle esta distinción en mi libro El temblor del ser: cuerpo $y$ afectividad en el pensamiento tardio de Martin Heidegger, Ed. Biblos, 2012, especialmente en el capítulo "Del entre al con", p. 108 ss. 
(Nancy, 2013, 31). La strucción expresa "la simultaneidad no coordinada de las cosas o los seres, la contingencia de su copertenencia, la dispersión de las abundancias de aspectos, especies, fuerzas, formas, tensiones y distensiones (instintos, pulsiones, impulsos). En esta abundancia ningún orden se hace valer por encima de los otros: ellos parecen todos condenados a meterse unos en los otros, a disolverse o a confundirse los unos en los otros" (Nancy, 2013, 34).

El tercer trazo importante a subrayar tiene relación con el otorgarles a los cuerpos el lugar y la función de constituir la apertura del espacio y con ello la condición de posibilidad de la existencia. Entre las generalizaciones más comunes a lo largo de la historia de la filosofía está la de adjudicar lo abierto a cierto carácter de las ideas y lo cerrado a los cuerpos; en Nancy los cuerpos son condición de posibilidad de la existencia, "dan lugar a la existencia" (corpus, p16) y en este sentido abren, y lo primero que abren, por decirlo de algún modo, es el espacio; es la categoría de espaciamiento, los cuerpos inauguran el espacio, espacían, son espaciosos y eso es una forma de apertura. "Los cuerpos no son de lo "pleno", del espacio lleno (el espacio está lleno por todas partes): son el espacio abierto, es decir, en algún sentido el espacio es más bien espacioso que espacial, o eso que se puede todavía llamar el lugar... un cuerpo es el lugar que abre (Corpus, p16 et 18 , tr. 15 y 17 ).

Hay en este punto algo muy relevante: se puede plantear un cierto momento ontológico de apertura en los cuerpos, desde su materialidad, que posee matices distintos a la apertura heideggeriana del Dasein. En primer lugar, en Nancy se trata de los cuerpos del mundo y no sólo de los humanos, en cambio en Heidegger la apertura en cuanto tal acontece en el Dasein, la existencia humana, y desde allí "se iluminan" las cosas.

En segundo lugar, y pensamos que esto tiene que ver directamente con la cuestión de la materialidad, en Heidegger el Dasein está abierto al mundo ante todo por habérselas con posibilidades, $y$ en este sentido su apertura es fundamentalmente temporal, el Dasein, el "ahí" del Dasein es un estar arrojado prioritariamente al tiempo, en cambio aquí habría un momento espacial en la apertura, o mejor dicho, acontecería la apertura del espacio propiamente tal en el estar unos con otros de los cuerpos. En un breve texto de su libro El peso de un pensamiento, titulado justamente "Espacio contra tiempo", Nancy apela a pensar hasta el pensamiento mismo desde la espaciosidad, “¿y si el pensamiento fuera espacioso?” (118) y a pensar el instante como una categoría más espaciosa que temporal, 
criticando la priorización exacerbada del tiempo a lo largo de la "historia" del pensamiento, llama a pensar el "espacio de tiempo como una apertura de un topos en el presente" (118).

Pues bien, la tesis central de este escrito es que esta apertura pensada junto a la relacionabilidad y modalidad de los cuerpos apuntan a un momento de la materialidad que podría ser pensado en un sentido amplio como libertad, en el sentido que los cuerpos no están absolutamente determinados y reducidos a un qué sino que exceden la determinación y por ello también la necesidad y este momento se corresponde con la lectura que realizan varios contemporáneos sobre el clinamen; es donde nos detendremos ahora.

\section{Materialidad de los cuerpos}

Queremos recalcar que estamos hablando de todos los cuerpos y no sólo de los cuerpos humanos. Por esto, el plantear un momento de libertad o apertura no puede entenderse asociándolo a un libre albedrío o a la voluntad. La pregunta entonces, y es lo que guiará a partir de ahora esta reflexión, es cómo se puede entender una libertad o una apertura "aplicable" a todos los cuerpos y no sólo a los humanos, o sea una libertad de los cuerpos en tanto que tales, desde su materialidad.

Varios autores franceses contemporáneos retomarán el antiguo tópico del clinamen o parénklisis iniciado con los atomistas griegos, específicamente con Epicuro, y reformulado luego por Lucrecio en De Rerum natura. Este tópico, en el que nos detendremos ahora, ha sido trabajado de forma intermitente pero continua a lo largo de la historia del pensamiento reluciendo en autores tan diversos como Giordano Bruno o el mismo Marx. Pero es a partir de 1970 que vuelve a aflorar con fuerza inusitada. Para acercarnos, aludiremos primero brevemente a la tesis clásica de la parénklisis o clinamen buscando los elementos que nos ayuden a articular una pregunta por el sentido que pueda tener la libertad o apertura de los cuerpos a partir de su materialidad; luego nos dirigiremos a la lectura del clinamen en algunos contemporáneos como Michel Serres, Luis Althusser, Jaques Derrida y Jean-Luc Nancy.

\subsection{Parénklisis o clinamen en Epicuro y Lucrecio:}

El atomismo de Epicuro tiene como base que "no hay algún ordenamiento impuesto por algún agente externo al proceso de la materia 
misma" (García Gual, 2002, 138), de hecho, solo habría átomos y espacio33. Los cuerpos serían aglomeraciones de átomos y conformarían un cosmos infinito que se compondría a su vez de infinitos cosmos.

Hay que tener por garantizado que hay también cosmos infinitos (kosmoiápeira), unos iguales a éste y otros distintos, pues los átomos al ser infinitos, como anteriormente se demostró, se desplazan a los lugares más apartados incluso, puesto que los átomos de esta naturaleza, con los que podría formarse o por medio de lo que podría ser constituido un mundo, no quedan agotados ni con un solo cosmos ni con cosmos limitados, ni con cuántos son como éstos ni con cuántos son diferentes a éstos. La consecuencia de ello es que no hay nada que impida la infinitud de los cosmos (Epicuro, Ed. Usener, fr. 45, 53s).

El asunto es que si aceptamos que el orden cósmico viene "desde adentro" o bien este orden estaría completamente determinado, o bien habría que "introducir" algún elemento o propiedad en la materia con cierta indeterminación, que sería precisamente el clinamen.

La primera tesis ha sido adjudicada tradicionalmente a Demócrito, y la segunda a Epicuro. Por ejemplo, el trabajo doctoral de Marx va en esa dirección y como afirma Ruiz, "es el primer filósofo que rescata de modo decidido la noción de clinamen en relación con su efectividad realizativa, tanto en el dominio de lo ético como en el de lo político" (Ruiz, 2011, 62). Ciertamente hay algunos que difieren de esa línea interpretativa, específicamente Wisman que argumenta la distorsión intencionada del pensamiento de Demócrito por parte de Aristóteles que es de donde proviene nuestra información de Demócrito. Aristóteles estaría recogiendo el pensamiento de los presocráticos para situarse él mismo como una cierta culminación, con lo que iría calificando y acomodando la exposición de los autores para aquel fin (Wisman, 2010, 8).

Deteniéndonos en la segunda tesis; observamos que si los cuerpos se componen de átomos debe haber algo en ellos que los haga colisionar y componerse o dividirse: Epicuro se refiere a una suerte de "espontaneidad interna” (García Gual, 2002, 140) o “causa intrínseca espontánea” (Oyarzún

3 Queda pendiente aquí la ineludible reflexión de la idea de átomo pensada por Epicuro y Demócrito, trabajo ya realizado en parte en el texto de M. Rodríguez que se va desplazando desde una lectura clásica del átomo como suficiencia sustancial a una especie de campo de efectuación. (Cf. Rodríguez M. Byzantion Nea Hellás no.33 Santiago nov. 2014, pp 101-118). 
y Molina, 2005, 9) llamada parénklisis ${ }^{4}$ según la cual los átomos se desvían mínimamente de su trayecto, posibilitando así sus colisiones y con ello la composición de los cuerpos.

Esta espontaneidad en la desviación es la que hace hablar de un movimiento "libre". En este sentido la desviación espontánea de los átomos si bien no niega el sentido de la necesidad y un orden determinado, al menos lo restringe, y por ahora es el primer sentido que podemos dar a esta apertura o libertad de los átomos.

Pero eso no es todo, pues "en todo tiempo y en todo lugar 'puede' ocurrir tal desviación” (Oyarzún y Molina, 2005), y encontramos aquí un segundo sentido de apertura o libertad vinculado al reino de la posibilidad que restringe el de la necesidad. "Puede afirmarse que el clinamen instaura, en el origen y a partir del origen, el reino de la "posibilidad" en coexistencia y coincidencia con el reino de la necesidad." (Oyarzún y Molina, 2005, 15). Es desde aquí que se puede afirmar la vinculación de la física y la ética (y la política) como lo afirman Oyarzún y Molina. Veremos que, yendo más allá de Epicuro, pero con él, las propuestas contemporáneas que trabajaremos a continuación afirmarán una confluencia física-ética-política aún más radical.

Escuchemos la formulación del clinamen en el texto de Lucrecio:

Cuando los cuerpos son llevados hacia abajo en línea recta a través de lo inane por sus propios pesos, aquellos, en un tiempo incierto y en lugares indeterminados, se desvían un poco de su curso, justo lo suficiente para que se pueda decir que el movimiento ha cambiado. Porque si ellos solieran declinar, todos, como gotas de lluvia, caerían de arriba abajo a través de la profundidad delo inane; ninguna colisión habría podido llegar a darse, ningún golpe habría podido producirse para los primeros principios: de modo que la naturaleza jamás habría creado nada (Lucr., II, 217-224).

Solamente dejemos consignada aquí la "definición" de clinamen como el desvío mínimo del curso de un átomo que permite las colisiones que conforman la naturaleza.

4 No hay registros del uso de este término en los textos conservados de Epicuro, aunque está más o menos consensuado que tiene que haber sido escrito y que ocupó un lugar importante en su sistema filosófico. 


\subsection{Lecturas contemporáneas del clinamen}

Ahora bien, la cuestión es preguntarse por qué hoy se retoma el clinamen, y por ahora podemos decir que hay un referente común respecto al cual, desde distintos ámbitos varios contemporáneos quieren diferir, y es lo que podríamos llamar materialismo científico de raigambre positivista.

Por una parte, Althusser y su crítica al materialismo dialéctico, que como tal no sería un verdadero materialismo, pero también por el lado de Serres y su crítica a la reducción de la física de Galileo, que si bien es verdadera representa otro horizonte físico distinto a aquel abierto por Lucrecio y que se emparentaría con experiencias científicas contemporáneas como las de Prigogine o Thom. Derrida, en la misma línea, se pregunta ¿cuál es la diferencia entre superstición o paranoia, por un lado, y ciencia, por otro, si todas están marcadas por una propensión compulsiva hacia la interpretación de los signos aleatorios para restituirles un sentido, una necesidad, una destinación? (Derrida, 1987, 19) y Nancy a través de la discusión con Heidegger quien divide a los entes entre los que tienen mundo, los que tienen poco y los que no tienen buscará la sustracción del carácter de necesidad al mundo en cuanto tal. El clinamen en todos estos autores es la herramienta que puede desmantelar la comprensión del mundo desde la pura necesidad y con ello la escisión de los entes que se rigen por la libertad y los que se rigen por la necesidad. El clinamen rompe o confunde la escisión entre materia y alma.

Habíamos desprendido de los textos de Epicuro y Lucrecio el enunciado "el clinamen es la desviación mínima del curso de un átomo". El sentido más preponderante otorgado al clinamen a partir de aquí es sin duda el de espontaneidad, trabajado también como aleatoriedad: dado un orden interno, en un tiempo y lugar indeterminado éste es necesariamente disuelto por un acontecimiento adventicio (Lezra, 2016), azaroso.

Justamente el texto de Derrida titulado "Mis chances" acude a Epicuro y Lucrecio para mostrar una "reserva de indeterminación respecto a cualquier cálculo posible" (Derrida, 1987, 4). Lo aleatorio impide o al menos restringe el principio mismo de la ciencia, ya sea en términos de encadenamientos causales ya sea en términos de previsibilidad.

Michel Serres varía levemente esta tesis, concibiendo el clinamen como desviación del equilibrio:

"Este es el genial golpe de la física de los átomos: no hay círculos, no hay más que torbellinos. No hay cierres exactos, no hay circunferencias puras, espirales desfasadas, erosionadas. El círculo degenera en una 
hélice cónica, el círculo pitagórico o platónico se convierte en helicoide arquimediana. En otras palabras, la naturaleza no está dotada de movimiento perpetuo.

No hay más que un flujo laminar. El mundo es multiplicidad de flujos, inclinados los unos en relación a los otros. Debido precisamente a la inclinación, estas circulaciones no describen círculos. Una circunsferencia más un ángulo, no importa cuán pequeño sea éste, produce una espiral" (Serres, 1977, 74).

Althusser hablará directamente de materialismo aleatorio, apelando a una corriente subterránea de este materialismo que habría transcurrido por siglos. "Para Althusser, aquello que importa es menos el desencadenamiento de leyes que el carácter aleatorio de la "toma de consistencia" (prise) de este encuentro que da lugar al hecho cumplido del cual se pueden enunciar las leyes" (Ibrahim, 2012, 11). El materialismo aleatorio es el de la contingencia y se opone al de la necesidad y teleología. "Puede decirse que el mundo es el hecho consumado, en el cual, una vez consumado el hecho, se instaura el reino de la Razón, del Sentido, de la Necesidad y del Fin. Pero la propia consumación del hecho no es más que puro efecto de la contingencia, ya que depende del encuentro aleatorio de los átomos debido a la desviación del clinamen" (Althusser, 1994, 34).

Hasta ahora hemos hablado indistintamente de espontaneidad, aleatoriedad, azar, probabilidad y es lo que hacen estos autores, aunque podríamos mencionar ciertos matices. Marcel Conche en su libro Lo aleatorio (2012) realiza un exhaustivo análisis donde justamente distingue estas acepciones, donde por ejemplo hay acontecimientos aleatorios que no ocurren por azar, porque no son fortuitos e inversamente hay acontecimientos fortuitos que no son aleatorios. Del mismo modo lo aleatorio no se identifica con lo probable, "lo aleatorio marca la duda (hésitation) entre dos posibles y el grado de incerteza. Lo probable implica una información en cuanto a las chances de realizar los diversos posibles... La noción de probabilidad marca una conquista sobre lo aleatorio; ella permite reducir, racionalizar lo aleatorio." (Conche, 2012,21). En la misma línea afirma Althusser "Nada decide, ningún principio de decisión decide por adelantado en esta alternativa" (39)

Lo aleatorio tiene que ver con la indeterminación e imprevisibilidad de ciertos acontecimientos y mientras algunos, por ejemplo la biología moderna, atribuyen esta imprevisibilidad al entorno, la teoría del clinamen lo atribuye al átomo. 
"La naturaleza, según Epicuro, es 'creadora' (Lucrecio habla de natura creatrix, I, 629; II 116; V, 1362), eso quiere decir que ella es libre respecto a sus propias producciones, condicionada pero en absoluto determinada por aquello que ella ha sido, siempre libre para lo nuevo, lo inédito. Sin el clinamen, no se podría explicar ni esta creación de la naturaleza que es el mundo, ni la libertad de los vivientes -libertad innovadora, creadora (cf. Lucrecio II, 251-262)".

Encontramos en esta última acepción un rasgo que se desvía un poco del curso tomado hasta ahora, hemos hablado de imprevisibilidad, indeterminación, no-necesidad, contingencia y ahora variamos hacia el carácter libre y creador del desvío. La postura de Nancy es cercana a esta tesis, pero a partir de ella se distancia de la lectura del azar. Nancy está buscando, en discusión con Heidegger, y asumiendo que es una de las cuestiones más difíciles y que no está resuelta y de la cual habrían de derivar una toda una ética y una política, una manera de no caer en la división del mundo entre lo humano y lo no humano, una comprensión de mundo como "una co-pertenencia esencial de la existencia con el existir de toda cosa" (Nancy, 1988, 202), (y aquí existencia es justa y acentuadamente diferente al Dasein) donde el clinamen entendido como la "libre abertura del hay" (Nancy, 1988, 202s), sería aquella juntura desde donde separarse de la necesidad. Escuchemos:

"Si se profundiza convenientemente en esta copertenencia esencial (de lo sin-esencia), se encontrará que ninguna cosa puede ser simplemente 'necesaria', y que el mundo no es 'necesario'. No se podrá aislar de un lado la causalidad de los fenómenos, del otro, la libertad noumenal ¿Qué se encontrará entonces? Intentemos, provisionalmente, decir: algo así como un clinamen, que no sería el azar (otra necesidad), sino la libre abertura del hay en general- que no es nunca general-precisamente, sino que está siempre en el orden del 'cada vez'.

Clinamen o declinación, inclinación del 'hay', del es gibt, de la ofrenda... en este sentido, la piedra es libre" (Nancy, 1993, 202s).

En esta apretada cita hay varias cuestiones, lo primero es que su modo de salir de la necesidad se separa, a diferencia de todos los otros autores contemporáneos mencionados aquí, del azar, y por ello de la lectura del clinamen desde lo aleatorio para dar paso a una cierta idea de libertad como una apertura material de las cosas existentes a partir del cual nosotras las cosas estamos vueltas las unas a las otras, inclinadas las unas a las otras y por eso hay mundo. "No se hace un mundo con simples átomos. Hace falta 
un clinamen. Hace falta una inclinación, o una inclinación del uno hacia el otro, del uno por el otro, del uno al otro" (Nancy, 1999, 17). Se trata aquí de una materialidad abierta que posibilita el juego e indeterminabilidad por exceso de los cuerpos, se trata de singularidades que difieren, que consisten en diferir-se, y eso correspondería a lo que los más antiguos llamaron clinamen. "La materia pertenece.... a la estructura del sentido del mundo. Así hay que releer en los democritianos la caída de los átomos en el vacío y el clinamen: el desvío y el contacto, el montaje, la separación, la tangencia el entre-dos y el choque recíproco del hay difractado singular. La singularidad es material...y recíprocamente la materia siempre es singular...la materia es el abrirse paso (le frayage) mismo o la fractalidad de los fragmentos, lugares y de lo que tiene lugar (avoir-lieux)" (Nancy, 1993, 97s).

En segundo lugar, es que al sustraer el carácter de necesario al mundo y otorgarles libertad a las cosas lo que está haciendo es justamente establecer una copertenencia material desde donde nos encontremos los cuerpos en forma simétrica y ya no desde la estructura del hombre libre que domina a una naturaleza que se rige por leyes necesarias sino desde nuestra apertura, nuestra exuberancia material. Esto está muy en la línea epicureana de confluencia de lo que hoy llamaríamos ámbito de la física y de la ética, o como afirman Pablo Orarzún y Eduardo Molina de "una ampliación del sentido mismo de lo físico" $(2005,92)$.

Un paso más, y que está presente de modo explícito en todos los autores a los que hemos aludido, y es que el clinamen es también una suerte de deseo y de placer; para ser más exactos, el clinamen y el encuentro son dos caras de una misma moneda, no podemos pensarlos como uno anterior a otro, es clinamen-encuentro y eso es el mundo. Si miramos la cara del encuentro, ella tiene rostro de placer.

Escuchemos a Derrida:

"Se trata de la lectura indeterminada de la palabra voluptas o voluntas: la simple diferencia de una letra introduce un clinamen ahí donde justamente Lucrecio explica por qué el clinamen es la condición de la libertad, de la voluntad o de la voluptuosidad que se arranca de esta forma al destino (fatisavolsa). En cualquier caso, el contexto no deja ninguna duda sobre la relación entre clinamen, libertad y placer. El clinamen del principio elemental, a saber el átomo, la ley del átomo, sería el principio de placer" (Derrida, 1987, 257). 
No en vano el poema de Lucrecio comienza con la invocación a Venus, ella es quien gobierna el poema, y de allí que Serres llegue a hablar de dos físicas completamente distintas, la de venus y la de marte, donde ambas son verdaderas y la física de venus "es una ciencia de las caricias. Los objetos a distancia se mandan besos y cambian sus pieles. En la lejanía está la torre cuadrada, angulosa, rígida, rugosa; se acerca a mí redonda, lisa y suave. Fenomenología de la caricia, saber voluptuoso" (Serres, 1977, 134).

Se trata de una libertad más voluptuosa que ilustrada, no se separa del deseo pues "la realidad del deseo, el efectivo impulso dado en el ejercicio de la voluntad, es correlativa a nivel atómico con la existencia plausible del clinamen, esa mínima desviación que habilita a pensar y a decir que ha habido un cambio de movimiento, que se ha emprendido una acción sobre las cosas" (Ruiz, 2009, 72).

Este saber nos lleva otra vez a Epicuro. Como en el Jardín, se trata de un saber de lo cercano a través del encuentro de los singulares, es el saber de la proximidad y el tacto, el saber del frote de los átomos. Epicuro, como es sabido, nos dirá que "el placer es el principio y fin de una vida afortunada (makarios zen)" (Epicuro, Ed. Usener, fr.128, 62.) y es que sólo desde el placer puedo conocer. "Pues no sé yo qué idea puedo hacerme del bien si suprimo los placeres del gusto, si suprimo los afrodisíacos, si suprimo los del canto y si suprimo los movimientos placenteros que de las formas bellas recibe la vista" (Epicuro, Usener, fr.67, 27).

La ética es una física, libertad y placer son la inclinación y el encuentro de las cosas. 


\section{Referencias bibliográficas:}

ALTHUSSER, L. (2002). "La corriente subterránea del materialismo del encuentro" en Para un materialismo aleatorio. Madrid: Arena Libros.

BULO, V. (2012). El temblor del ser: cuerpo y afectividad en el pensamiento tardio de Martin Heidegger. Buenos Aires: Ed. Biblos.

CONCHE, M. (2012). L'aléatoire, París: Les belles lettres.

DERRIDA, (1987). "Mes chances. Au rendez-vous de quelques stéréophonies épicuriennes", en Psyché Tome I, París: Galilée.

HERMANNVS VSENER (1966). Epicvrea,Stuttgard: Verlag B.G. Teubner Stuttgart.

GARCÍA GUAL, C. (2002), Epicuro. Madrid: Alianza.

IBRAHIM, A. (2012), Avantpropós, en aoutur de Althusser, penser un materialisme aleatoire: problemas et perspectives, París: Ed. Le temps de crises.

LEZRA J. (2016). Lucretius and Modernity: Epicurean Encounters Across Time and Disciplines: Ed, New Antiquity.

LUCRECIO, (1997) De rerum natura, Ed. Crítica y versión rítmica Agustín García Calvo, Madrid: Ed. Lucina.

OYARZÚN, P. Y MOLINA, E. (2005). “Sobre el clinamen”, en Méthexis18, pp. 67-87.

OYARZÚN, P. (2011). "La integración alma-cuerpo en Epicuro", en Estudios, $\mathrm{n}^{\circ} 13,11-16$.

NANCY, J.L. (2013). Archivida, Trad. V. Bulo y M. Bardet, Buenos Aires: Quadrata.

--, (2006). Corpus. Paris: Métailié.

-, (2007). El peso de un pensamiento, Castellón: Eliago Ediciones.

-, (1999). La común auté dés oeuvrée, Paris: Christian Bourgois Editeur.

, (1988). L' expérience de la liberté, Paris: Galilée

, (1993). Le sens du monde. Paris: Galilée.

RODRÍGUEZ, M. (2014). "Demócrito: una 'nueva' práctica de la filosofía”, en Byzantion Nea Hellás No33 Santiago nov., pp 101-118.

RUIZ, M. "El clinamen, el animal y la virtud", en http://www.biopolitica.cl/docs/ MRuiz_Clinamen_animal_virtus.pdf.

--------, (2009). "La política De rerum natura. Efectos del clinamen en la idea de comunidad de Lucrecio, en Atenea 500, pp. 41-54.

---------, (2011). "Lucrecio y el acontecimiento. Experiencia de comunidad y comunidad de experiencia", en Política y acontecimiento, Santiago: FCE.

SERRES, M. (1977). La naissance de la Physique dans le text de Lucrèce, Paris: Les Éditions de Minuit.

WISMAN H. (2010). Les avatars du vide: Démocrite et les fondements de l'atomisme, Paris: Editions Hermann. 